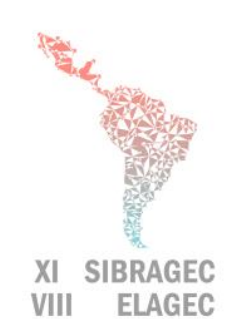

VIII ELAGEC

\section{SIMPÓSIO BRASILEIRO DE GESTÃO E ECONOMIA DA CONSTRUÇÃO}

VIII ENCUENTRO LATINOAMERICANO DE GESTIÓN

Y ECONOMÍA DE LA CONSTRUCCIÓN

Do conhecimento à ação: práticas avançadas de gestão da produção

Londrina, Paraná, Brasil. 23 a 25 de Outubro de 2019

\title{
ROTINA DE PROGRAMAÇÃO PARA GERAÇÃO DE MODELOS BIM VISANDO ESTIMATIVAS DE CUSTOS
}

\author{
ARAÚJO, C. S. (1); SIQUEIRA, L. C. (2); BRITO, B. L. (3); FERREIRA, E. A. M. (4) \\ (1) UFBA, (71)99237-1088, e-mail: araujo.caroline92@gmail.com, (2) UFBA, e-mail: \\ leandrocandidosiq@gmail.com, (3) UFBA, e-mail: leaodebrito@gmail.com, (4) UFBA, e-mail: \\ ferreira.eam@gmail.com
}

\begin{abstract}
This study presents a comparison between a general programming routine and a reference routine from the literature. Both routines propose a cost estimate in the initial phases of the project using generative programming associated to BIM, which involves generating a model and extracting quantitative data through Dynamo, Revit and Excel software. The purpose of this study is to refine the reference routine to allow variations in the construction model by modifying only the data inputs, without having to change the programming structure for each new modeled solution. The research strategy adopted in this work is Design Science Research (DSR), which is oriented to the solution of relevant and pragmatic real-world problems. It has been divided into the stages of awareness, suggestion, development, evaluation and completion. In order to compare the results, the structural elements (beams, slabs and pillars) of a building were modeled through the general routine. The routine presented an error of $12 \%$ in relation to the budgeted cost and was considered a viable and fast solution for the creation of BIM models for preliminary cost estimates, in order to support decisions in the initial phases of the project.
\end{abstract}

Keywords: BIM, Cost Estimation, Generative Programming.

\section{INTRODUÇÃ̃O}

Em fases iniciais de projeto os elementos que compõem o custo não se encontram detalhados de forma suficiente, dificultando a elaboração de estimativas de custo precisas (LOSSO, 1995).

O estudo de custos da construção, no entanto, deve acompanhar todo o desenvolvimento do projeto desde o seu princípio, considerando que serve de apoio para a tomada de decisão e para análises de viabilidade (LOSSO, 1995; GONÇALVES, 2011).

Segundo Kern (2005), a maior influência na redução do custo total ocorre na etapa de concepção de um empreendimento. Para compreender os impactos resultantes das decisões tomadas nessas fases iniciais faz-se necessária a capacidade de estimar os custos adequadamente (LIMA, 2013).

Como meio de realizar tais estimativas, diversos métodos foram desenvolvidos, entre eles o Custo Unitário Básico (CUB), que utiliza parâmetros de custo por metro quadrado para determinação do valor total da edificação, e o Custo Unitário Geométrico (CUG), que utiliza características geométricas da edificação. No entanto esses métodos apresentam baixa precisão nas estimativas, tendo sido relatadas variações de 31 a $45 \%$ 


\section{SIBRAGEC - ELAGEC 2019 - de 23 a 25 de Outubro - LONDRINA - PR}

com uso do CUB e de 9 a $23 \%$ com uso do CUG em estudos realizados por Lima et al. (2016).

Segundo Ávila, Librelotto e Lopes (2003), o erro na avaliação do custo pode variar em torno de $15 \%$ a $20 \%$ nas estimativas feitas em fases de anteprojeto. No presente trabalho, esta margem de variação é tomada como referência para avaliar a precisão dos valores obtidos.

Considerando este contexto, a Modelagem da Informação da Construção (BIM) apresenta-se como uma ferramenta que possibilita a simultaneidade entre estimativa e desenvolvimento do projeto, contribuindo para o conhecimento antecipado das implicações de tomadas de decisão (LIMA, 2013). Outro aspecto importante é o fato do uso do BIM proporcionar uma quantificação automática e precisa, aumentando a velocidade da estimativa e permitindo que diferentes alternativas de projeto sejam exploradas, levando à possibilidade de maior controle no dimensionamento dos recursos da obra (SANTOS, 2009).

Além disso, com o BIM foram introduzidas novas ferramentas e softwares para projetistas, que possibilitam a criação de algoritmos para automatizar tarefas que consomem muito tempo quando executadas manualmente (ANDRADE, 2017). Existem aplicativos que possibilitam a utilização de programação generativa para criação de modelos virtuais, como é o caso do Dynamo, um software da Autodesk que permite o desenvolvimento de rotinas utilizando programação visual (ANDRADE, 2017; SILVA et al., 2018). Por meio dele, o usuário faz uso de "nós" (pequenos blocos de código) que permitem visualizar as alterações no fluxo de dados de forma constante, facilitando o processo de programação.

Dentro desse cenário, Brito (2018) propôs um método para determinação de estimativas de custo a partir de programação generativa associada ao BIM em fases iniciais do projeto. O método supracitado apresentou o desenvolvimento de modelos de alguns empreendimentos para análises de custo, no entanto, para cada novo empreendimento estudado eram necessários ajustes na rotina original ou a criação de uma nova rotina. Essa característica implica na necessidade de que o projetista tenha conhecimentos específicos relacionados à programação, além de ter como possível consequência o aumento do tempo gasto na etapa de modelagem.

Considerando esta lacuna, o presente estudo visa dar continuidade ao trabalho de Brito (2018) com base nas suas recomendações para trabalhos futuros, tendo como objetivo principal aperfeiçoar a programação para permitir que sejam feitas modificações no modelo da edificação através da variação dos parâmetros de entrada, sem que seja necessário alterar a estrutura da programação para cada nova solução modelada.

\section{MODELAGEM DA INFORMAÇÃO DA CONSTRUÇÃO}

BIM é uma tecnologia que possibilita a representação do processo de construção em um modelo digital que contém geometria e dados precisos, além de permitir a interoperabilidade entre softwares, tornando o processo de projeto e construção mais integrados (ZHANG et al., 2013).

Algumas aplicações e vantagens de um modelo BIM são: visualização precisa, facilitando a interação com o usuário; melhor design e processos mais rápidos e eficazes, possibilitando que propostas de construção sejam rigorosamente analisadas e simuladas rapidamente, além de permitir estimativas de custos mais rápidas, já que 
SIBRAGEC - ELAGEC 2019 - de 23 a 25 de Outubro - LONDRINA - PR

quantidades de material são automaticamente extraídas e alteradas com a modificação do modelo (AZHAR et al., 2008).

\section{ALGORITMOS GENERATIVOS E O DYNAMO}

Algoritmos generativos podem ser definidos como um processo interativo que procura melhorar uma condição. Cada nova interação é uma melhoria comparada com a anterior, em um processo que se repete até que uma condição otimizada tenha sido atingida (KENSEK; NOBLE, 2014). Um exemplo é a transformação de dados de entrada em geometrias.

O uso de algoritmos generativos permite que o projetista crie conexões geométricas e matemáticas por meio de um software que permitirá a visualização em tempo real desses parâmetros (PINTO; PUPO, 2015).

O Dynamo, software de programação visual, funciona como um plug-in do Revit, permitindo que o projetista crie uma lógica visual para explorar projetos conceituais paramétricos e automatizar tarefas (Figura 1). Essa ligação possibilita que tarefas complexas que utilizem dados de famílias do Revit e encadeamentos lógicos sejam realizadas, auxiliando nos processos de escolha de soluções já nas etapas iniciais de desenvolvimento do projeto (SILVA et al., 2018).

Figura 1- Exemplo de programação visual no Dynamo: criação de polígonos a partir de parâmetros de entrada.

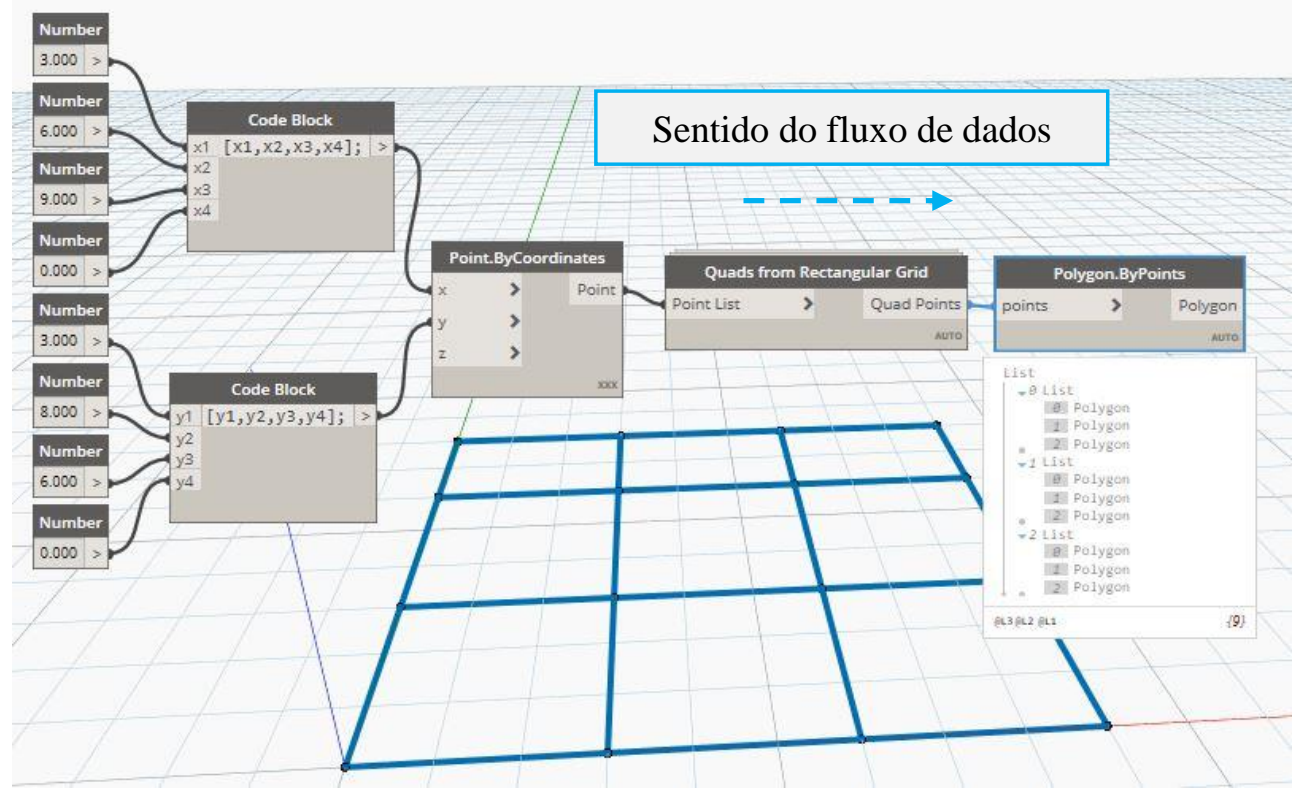

Fonte: Autores (2019).

\section{METODOLOGIA}

A estratégia de pesquisa adotada neste trabalho é a Design Science Research (DSR), que é orientada à solução de problemas relevantes e pragmáticos do mundo real (VAN AKEN, 2005). As fases de pesquisa estão indicadas na Figura 2, onde também está detalhado o produto da fase de desenvolvimento, que envolve o processo de aplicação da rotina geral a partir do projeto arquitetônico para obtenção do modelo BIM no Revit e da estimativa preliminar de custos. 
SIBRAGEC - ELAGEC 2019 - de 23 a 25 de Outubro - LONDRINA - PR

Figura 2- Fases da pesquisa e produto da fase de desenvolvimento.

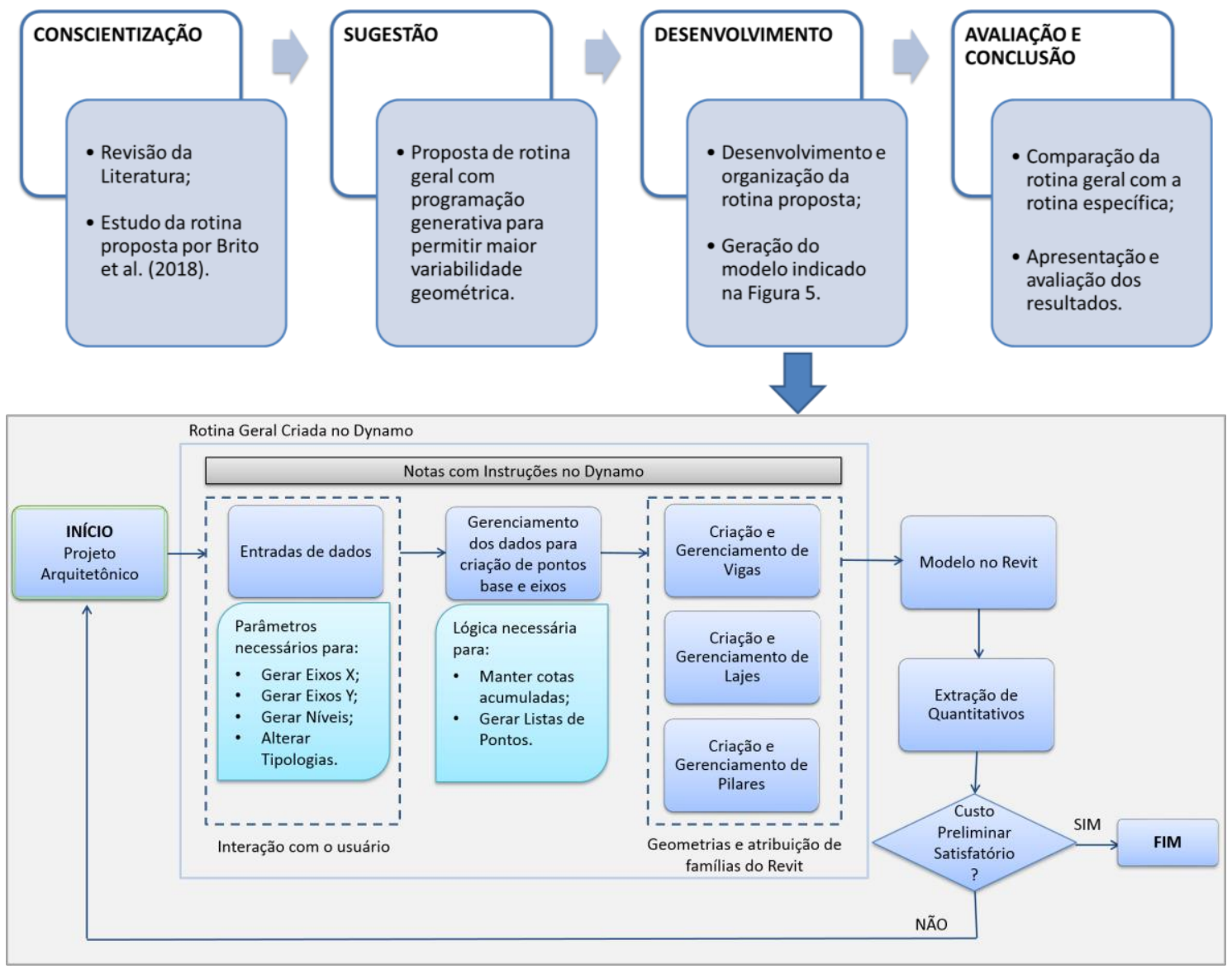

Fonte: Autores (2019).

A rotina geral foi desenvolvida no Dynamo tendo como base a Estrutura Lógica para Programação generativa de edifícios proposta por Brito et al. (2018), que foi aperfeiçoada para permitir a criação de diferentes modelos apenas alterando-se as entradas e sem modificar a estrutura da programação (Figura 3). Para fins de comparação de resultados, os elementos estruturais (vigas, lajes e pilares) do edifício indicado por Brito et al. (2018) no seu Estudo de Caso 4 foram modelados através da rotina geral.

Figura 3 - Demonstração de variabilidade de tipologias.
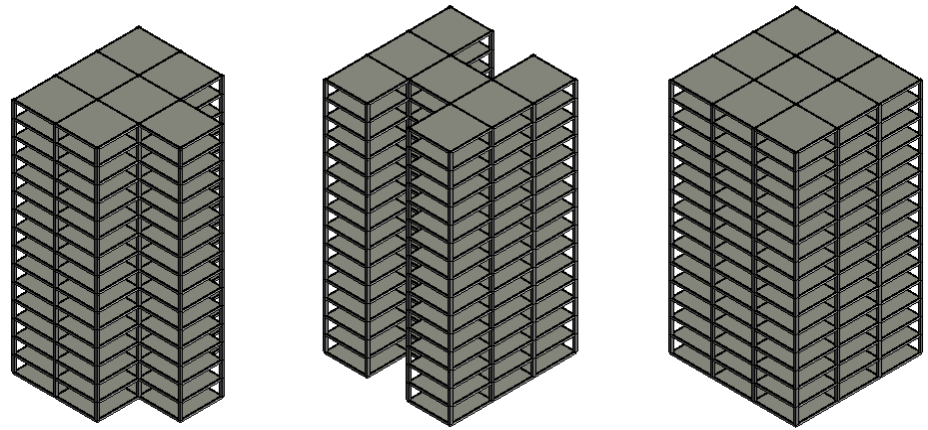

Fonte: Autores (2019). 


\section{ESTUDO EXPERIMENTAL}

A criação da rotina foi dividida em grupos principais, conforme representado na Figura 2. Na Figura 4 estes grupos estão indicados no Dynamo: a) parâmetros de entrada, onde o usuário fornece as diretrizes para criação da geometria, gerada através de coordenadas bidimensionais e tridimensionais; b) gerenciamento dos dados, onde as diretrizes dão origem aos pontos que servirão de base para as outras etapas; c) geração de vigas, onde a geometria das vigas é criada e o usuário pode decidir remover algum elemento do modelo, se necessário; d) geração das lajes e e) geração dos pilares, ambos seguindo o mesmo raciocínio das vigas. Há também um grupo contendo instruções para facilitar a utilização do programa pelo usuário, além de notas de texto explicativas espalhadas pelo programa.

Figura 4 - Rotina geral no Dynamo.

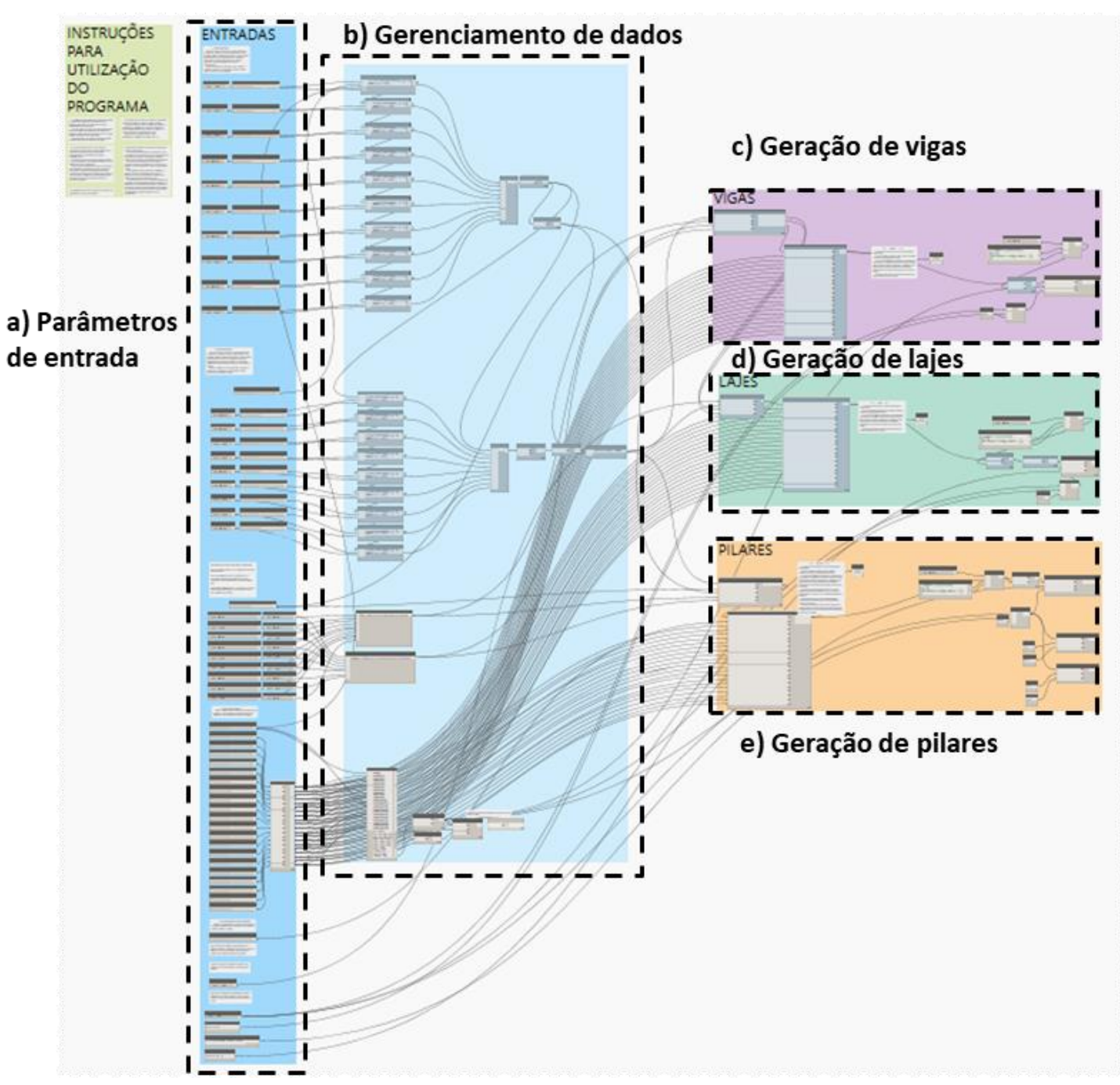

Fonte: Autores (2019).

Algumas etapas da programação foram aperfeiçoadas para melhorar as relações entre elementos e permitir a variabilidade da rotina: a) inserção de booleanos, nodes que assumem valores de verdadeiro ou falso, para que o usuário possa decidir entre duas opções, como apagar algumas vigas ou não; b) utilização de booleanos para que o programa seja executado em etapas, de acordo com o avanço na utilização da rotina, exigindo menor tempo de processamento do computador; c) mudança na maneira de 
SIBRAGEC - ELAGEC 2019 - de 23 a 25 de Outubro - LONDRINA - PR

criar os pontos base, transladando sempre o ponto anterior ao invés da criação ponto a ponto; d) incremento da lógica que translada geometrias em um determinado eixo, permitindo a alteração da tipologia, também com booleanos; e) utilização de nós que filtram valores nulos e listas vazias, para melhorar o tratamento dos dados e f) criação de nós personalizados, para diminuir o volume de nós na tela e melhorar a fluidez do programa.

Uma vez finalizada a programação da rotina, foram inseridos os parâmetros de entrada do empreendimento escolhido, dando origem ao modelo BIM no Revit, de onde foram extraídos os quantitativos (Figura 5). Em seguida, o custo foi estimado e comparado com os resultados do modelo alcançados por Brito et al. (2018) (Figura 6). Tais resultados foram atingidos utilizando os mesmos dados de custo unitário e processos para determinação do custo propostos por Brito (2018).

\section{Figura 5 - Modelo BIM pela rotina geral.}

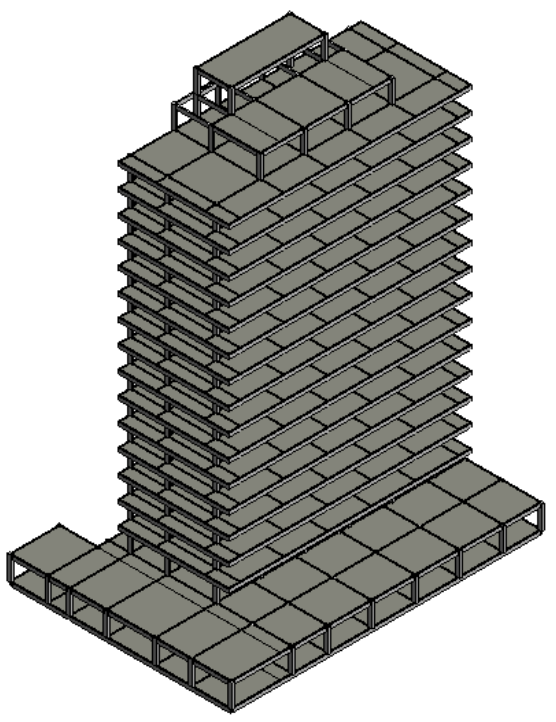

Fonte: Autores (2019).

\section{Figura 6 - Modelo BIM pela rotina específica.}

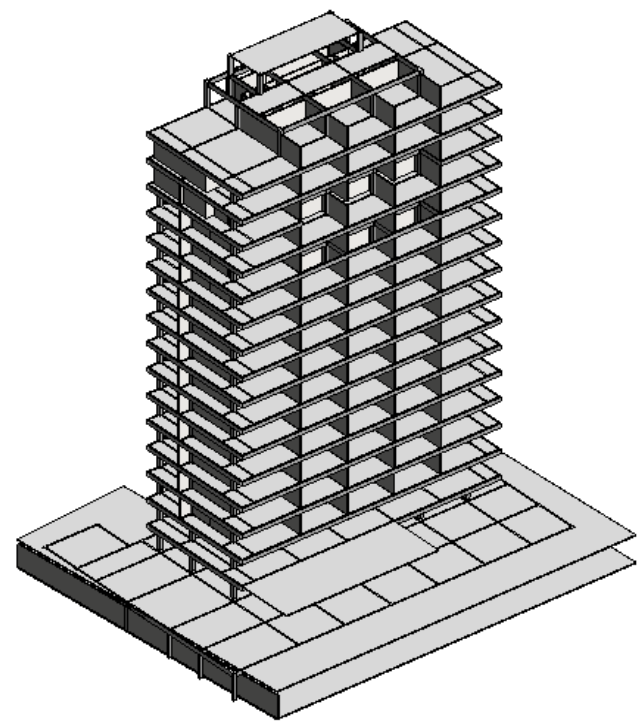

Fonte: Brito et al. (2018).

No Quadro 1 é apresentado o custo estimado para a etapa da superestrutura pela rotina geral e pela rotina específica, que foram comparados com o custo orçado pela construtora, utilizado como referência. Os respectivos erros percentuais foram calculados para comparar os resultados e verificar a acurácia alcançada.

\section{Quadro 1 - Comparativo dos Quantitativos}

\begin{tabular}{|c|c|c|c|}
\hline \multirow{2}{*}{ Rotina } & \multicolumn{2}{|c|}{ Superestrutura } & \multirow{2}{*}{ Erro Percentual } \\
\cline { 2 - 3 } & Custo orçado & Custo estimado & \\
\hline Geral & \multirow{2}{*}{$\mathrm{R} \$ 1.882 .145,65$} & $\mathrm{R} \$ 1.654 .938,47$ & $12 \%$ \\
\cline { 3 - 3 } & & $\mathrm{R} \$ 1.770 .916,45$ & $6 \%$ \\
\hline Específica & &
\end{tabular}

Fonte: Autores (2019). 
Para obtenção de toda a forma do edifício sem a necessidade de apagar muitos elementos ou alterar a rotina, a mesma foi executada quatro vezes, uma para cada variação na configuração do pavimento (Garagem, Pavimento Tipo, Cobertura e Caixa d'água), com a inserção dos respectivos deslocamentos em relação ao plano de origem. Para efeitos de análise, foram registrados os tempos gastos na modelagem e processamento de cada etapa na rotina geral e o tempo total para a criação do modelo BIM (Quadro 2).

Quadro 2 - Tempo de Modelagem e Execução (hh:mm:ss)

\begin{tabular}{|c|c|c|c|}
\hline Etapa & $\begin{array}{c}\text { Tempo de } \\
\text { Modelagem }\end{array}$ & $\begin{array}{c}\text { Geração da } \\
\text { Geometria no Revit }\end{array}$ & Total \\
\hline Garagem & $10: 30$ & $00: 46$ & $11: 16$ \\
\hline Pavimento Tipo (16) & $37: 50$ & $02: 13$ & $40: 03$ \\
\hline Cobertura & $13: 30$ & $00: 53$ & $14: 23$ \\
\hline Caixa d'água & $04: 00$ & $00: 50$ & $04: 50$ \\
\hline Estrutura Completa (soma) & $1: 05: 50$ & $02: 48$ & $01: 08: 38$ \\
\hline
\end{tabular}

Fonte: Autores (2019).

Considerando que para a utilização da rotina geral não são necessárias alterações na programação a cada nova modelagem proposta, o tempo gasto nessa etapa é eliminado em relação ao processo concebido por Brito et al. (2018). No entanto, o erro percentual é relativamente maior, já que os detalhes específicos de cada projeto não estão previstos na programação.

\section{CONSIDERAÇÕES FINAIS}

Levando em consideração a agilidade na etapa de modelagem, o erro percentual obtido e a comparação com a margem de erro aceitável de 15 a $20 \%$ apontada por Ávila, Librelotto e Lopes (2003), a rotina de programação proposta apresenta-se como uma solução viável para criação de modelos BIM para estimativas preliminares de custo, visando apoiar decisões em fases iniciais de projeto.

Com base na análise do processo de programação, soluções muito complexas ou incomuns continuam sendo uma limitação para a utilização de algoritmos generativos, em detrimento do tamanho do código, do alto tempo de processamento e demanda do computador. Em caso de soluções mais gerais, como no presente estudo, o algoritmo apresenta-se como uma possibilidade a ser considerada. Vale ressaltar que, algumas alterações e aprimoramentos na rotina podem levar a uma precisão maior e experiência melhor por parte do usuário, como a inclusão das alvenarias e das estruturas de delimitação do terreno.

Foi observado também que as estruturas de programação apresentadas neste trabalho podem ser direcionadas para diferentes etapas do desenvolvimento de projeto. A programação geral seria de grande valia na etapa de estudo de viabilidade, visto a rapidez e qualidade dos dados obtidos, possibilitando a análise de diversas soluções. Já a programação específica seria mais indicada para etapas de concepção projetual, onde o projetista precisa validar e analisar a forma do edifício de maneira mais precisa. 


\section{SIBRAGEC - ELAGEC 2019 - de 23 a 25 de Outubro - LONDRINA - PR}

Desse modo, conclui-se que o uso de programação generativa se apresenta como uma grande ferramenta, quando associada ao BIM, para realização de estimativas de custos, podendo apoiar decisões de projeto em diversas etapas.

\section{REFERÊNCIAS}

ANDRADE, L. S. de. Algoritmo para Alvenaria Estrutural em um Sistema BIM, 2017. Tese (Doutorado em Arquitetura e Urbanismo) - Programa de Pós-Graduação em Arquitetura e Urbanismo da Universidade de Brasília, Brasília, 2017.

ÁVILA, A.V., LIBRELOTTO, L.I., LOPES, O.C., Orçamento de Obras, Universidade do Sul de Santa Catarina - Curso de Arquitetura e Urbanismo - Planejamento e Gerenciamento de Obras, 2003

AZHAR et al. Building information modeling (BIM): A new paradigm for visual interactive modeling and simulation for construction projects. In: First International Conference on Construction in Developing Countries, 1., 2008, Karachi. Proceedings... Karachi: ICCIDC-I, 2008. p. 435-446.

BRITO, B. L. Estimativas de custo em fases iniciais de projetos a partir de modelos BIM e programação generativa: 2018. Dissertação (Mestrado em Engenharia Civil) - Programa de Pós-graduação em Engenharia Civil, Universidade Federal da Bahia, Salvador, 2018.

BRITO, B. L. de; ARAÚJO, C. S.; FERREIRA, E. A. M.; COSTA, D. B. Estrutura lógica para programação generativa em BIM aplicada a estimativas preliminares de custo. In: Encontro Nacional de Tecnologia do Ambiente Construído, 17., 2018, Foz do Iguaçu. Anais... Porto Alegre: ANTAC, 2018.

GONÇALVES, C. M. M. Método para gestão do custo da construção no processo de projeto de edificações: 2011. 182 f. Dissertação (Mestrado em Engenharia) - Escola Politécnica, Universidade São Paulo, São Paulo, 2011.

KENSEK, K. M.; NOBLE, D. E. Building Information Modeling: BIM in current and future practice. 1. ed. New Jersey, USA: John Wiley \& Sons, 2014. 397p.

KERN, A. P. Proposta de um modelo de planejamento e controle de custos de empreendimentos de construção: 2005. Tese (Doutorado em Engenharia) - Programa de PósGraduação em Engenharia Civil, Universidade Federal do Rio Grande do Sul, Porto Alegre, 2005.

LIMA, F. S. A. Custo unitário geométrico: uma proposta de método de estimativa de custos na fase preliminar do projeto de edificações: 2013. Dissertação (Mestrado em Engenharia de Produção) - Programa de Pós-graduação em Engenharia de Produção, Universidade Federal do Rio de Janeiro, Rio de Janeiro, 2013.

LIMA, M. F. de et al. Orçamento na fase da viabilidade: comparativo dos parâmetros custo unitário básico e custo unitário geométrico. In: Encontro Nacional de Tecnologia do Ambiente Construído, 16., 2016, São Paulo. Anais... . São Paulo: ENTAC, 2016. p. 3924 - 3937.

LOSSO, I. R. Utilização das características geométricas da edificação na elaboração de estimativas preliminares de custos: estudo de caso em uma empesa de construção. 1995. 146p. Dissertação (Mestrado em Engenharia Civil) - Programa de Pós-graduação em Engenharia Civil, Universidade Federal de Santa Catarina, Florianópolis, 1995.

PINTO, Y. A.; PUPO, R. Explorando a ferramenta de programação em design: um estudo sobre grasshopper. In: Congresso da Sociedade Ibero-americana de Gráfica Digital, 19., 2015, Florianópolis. Anais... São Paulo: Blucher, 2015. p. 686-690.

SANTOS, A. P. L. et al. A utilização do BIM em projetos de Construção Civil. Iberoamerican Journal of Industrial Engeneering (IJIE): Florianópolis, v. 1, n. 2, p. 24-42, 2009. 
SIBRAGEC - ELAGEC 2019 - de 23 a 25 de Outubro - LONDRINA - PR

SILVA et al. Designers of the XXI century: BIM software programming and the development of new competencies. In: Conference of the Iberoamerican Society of Digital Graphics, 22., 2018. São Paulo. Proceedings... São Paulo: Blucher, 2018. p. 538-545.

VAN AKEN, J. E. Management research as a design science: articulating the research products of mode 2 knowledge production in management. British Journal of Management, v. 16, p. 19-36, 2005. Disponível em: 〈https://doi.org/10.1111/j.1467-8551.2005.00437.x >. Acesso em: 18 abr. 2019.

ZHANG et al. Building Information Modeling (BIM) and Safety: Automatic Safety Checking of Construction Models and Schedules. Automation in Construction, v. 29, p. 183-195, 2013. 